\title{
Cramér-Rao Bounds for SNR Estimation of Oversampled Linearly Modulated Signals
}

\author{
Roberto López-Valcarce, Member, IEEE, Javier Villares, Member, IEEE, Jaume Riba, Senior Member, IEEE, \\ Wilfried Gappmair, Member, IEEE, and Carlos Mosquera, Member, IEEE
}

\begin{abstract}
Most Signal-to-noise ratio (SNR) estimators use the receiver matched filter output sampled at the symbol rate, an approach which does not preserve all information in the analog waveform due to aliasing. Thus, it is relevant to ask whether avoiding aliasing could improve SNR estimation. To this end, we compute the corresponding data-aided (DA) and non-data-aided (NDA) Cramér-Rao bounds (CRBs). We adopt a novel dual filter framework, which is shown to be information-preserving under suitable conditions and considerably simplifies the analysis. It is shown that the CRB can be substantially reduced by exploiting any available excess bandwidth, depending on the modulation scheme, the SNR range, and the estimator (DA or NDA) type.
\end{abstract}

Index Terms-Signal to noise ratio, Cramer-Rao bounds, oversampling.

\section{INTRODUCTION}

Communication receivers incorporate a variety of parameter estimators required for reliable data detection [1], [2]. With linear modulation schemes it is common to find estimators of phase and frequency offsets, channel gain, signal-to-noise ratio (SNR), etc., based on the output of the receiver Matched Filter (MF), sampled at the symbol rate. Although this introduces aliasing (unless the excess bandwidth is zero), the rationale for such approach is that in additive white Gaussian noise channels these samples provide sufficient statistics for data detection purposes. An exception to this rule is symbol timing estimation, which requires alias-free samples. Even assuming known symbol timing, it is not clear whether oversampling (with respect to the symbol rate) the received signal could improve estimation performance for any of the remaining parameters of interest, and if so, how to quantify such improvement.

Answering the above question for the problem of SNR estimation is the focus of this paper. Many techniques for managing communication resources, e.g., power control, adaptive modulation, turbo decoding, etc., are based on SNR knowledge [3]-[8]. A number of data-aided (DA) and nondata-aided (NDA) SNR estimators exist, most of them based

R. López-Valcarce and C. Mosquera are with the Signal Theory and Communications Department, University of Vigo, Vigo, Spain (e-mail: \{valcarce,mosquera\}@gts.uvigo.es).

J. Villares and J. Riba are with the Signal Theory and Communications Department, Technical University of Catalonia (UPC), Barcelona, Spain (email: \{jaume.riba,javier.villares\}@upc.edu).

W. Gappmair is with the Institute of Communication Networks and Satellite Communications, Graz University of Technology, Austria (e-mail: gappmair@tugraz.at).

Work supported by the Spanish Government and the European Regional Development Fund (ERDF) under projects TACTICA, COMPASS (TEC201347020-C2-1/2-R) and COMONSENS (CSD2008-00010), and by the Galician Regional Government under projects AtlantTIC, "Consolidation of Research Units" (GRC2013/009) and REdTEIC (R2014/037). on the symbol-rate sampled MF output; they can be generally grouped as either Maximum Likelihood (ML) [3], [9]-[13] or moments-based [9], [14]-[18]. Extensions to single- and multiple-input multiple-output channels also exist [19]-[21].

A few authors have considered SNR estimation with oversampling. Seeking robustness to symbol timing uncertainty, moments-type NDA SNR estimators based on the oversampled MF output were proposed in [22]. The DA ML SNR estimator and corresponding Cramér-Rao Bound (CRB) based on the oversampled signal at the MF input, with arbitrary oversampling factor, were given in [9]. In this model, the signal-free bandwidth available for estimation of the noise variance becomes arbitrarily large as the oversampling factor is increased. In contrast, the NDA estimator in [23] is based on secondorder statistics of the oversampled MF input, but assuming an antialiasing filter removing spectral content outside the signal bandwidth. This seems reasonable, as in practice such content is subject to interference from adjacent channels. Whereas the CRB for symbol-rate SNR estimation has been developed under a variety of settings [24]-[28], to our knowledge no results other than [9] are available for the oversampled case.

We develop the CRB for the oversampled case, under the practical constraint that only the useful signal band be processed. The CRB is obtained for (i) the DA case, (ii) the general NDA case, and (iii) the NDA case for second-order based estimators. Comparison with the corresponding symbolrate $\mathrm{CRB}$ is provided whenever possible. Since directly dealing with the oversampled MF output is cumbersome, we introduce a Spectrum-Derivative Matched Filter (SDMF) such that the MF and SDMF outputs, both sampled at the symbol rate, preserve all information about the original analog signal. It is found that this additional information (SDMF output samples) does reduce the CRB for SNR estimation, more so as the amount of excess bandwidth increases, and depending on the class of estimator considered (e.g., DA vs. NDA).

The paper is structured as follows. The system model is given in Sec. II. Sec. III presents the dual filter framework. The DA and NDA CRB are developed in Secs. IV and V. Numerical examples are given in Sec. VI, and Sec.VII draws some conclusions. All proofs are relegated to the Appendices.

Notation: vectors and matrices are represented by bold lowercase and bold uppercase symbols, respectively. $X(f)$ denotes the Fourier transform (FT) of $x(t)$, and ' $\star$ ' denotes convolution. By $\boldsymbol{x} \sim \mathcal{C} \mathcal{N}(\boldsymbol{\mu}, \boldsymbol{\Sigma})$ we mean that $\boldsymbol{x}$ is circular complex Gaussian distributed with mean $\boldsymbol{\mu}$ and covariance $\boldsymbol{\Sigma}$. $\operatorname{diag}\{\boldsymbol{x}\}$ denotes a diagonal matrix with the elements of $\boldsymbol{x}$ on its diagonal. For $\boldsymbol{A} \in \mathbb{C}^{m \times n}, \operatorname{vec}(\boldsymbol{A})$ denotes the $m n \times 1$ 
vector obtained by stacking the columns of $\boldsymbol{A}$. The $k \times k$ identity matrix is denoted by $\boldsymbol{I}_{k}$, and $\operatorname{tr}\{\boldsymbol{B}\}$ is the trace of $\boldsymbol{B}$.

\section{SYSTEM MODEL}

We consider single-input single-output communication systems employing linear modulation. Assuming a frequency-flat channel, the complex envelope of the available signal is

$$
r(t)=\sqrt{S} \mathrm{e}^{\jmath(2 \pi F t+\psi)} \sum_{l} a_{l} g_{T}(t-l T-\varepsilon T)+\sqrt{N} n(t),
$$

where $\left\{a_{l}\right\}$ are the unit-power transmitted symbols, and $g_{T}(t)$ is a square-root raised cosine (SRRC) pulse [1] with rolloff $\alpha \in[0,1]$ and symbol rate $1 / T$, normalized so that $\int_{-\infty}^{\infty} g_{T}^{2}(t) \mathrm{d} t=1$. The frequency, phase, and timing offsets are denoted by $F, \psi, \varepsilon$ respectively. It is assumed that $F$ and $\varepsilon$ are known; hence we will make in the sequel $F=\varepsilon=0$ w.l.o.g. As in [23], it is assumed that the received signal has been processed by an ideal lowpass filter with cutoff frequency $\frac{1+\alpha}{2 T}$ to produce $r(t)$. In this way, estimation is constrained to using only the information available within the useful signal band. Hence, the noise process $n(t)$ is assumed zero-mean, circularly symmetric Gaussian with a bandlimited power spectral density (psd) $S_{n}(f)=1$ for $|f| \leq \frac{1+\alpha}{2 T}$ and zero elsewhere.

The signal-to-noise ratio is defined ${ }^{1}$ as $\rho \triangleq S / N$. Since the main focus is on SNR estimation, it is useful to rewrite (1) in terms of $\rho$ (and after making $\varepsilon=F=0$ ) as

$$
r(t)=\sqrt{\rho N} \mathrm{e}^{\jmath \psi} \sum_{l} a_{l} g_{T}(t-l T)+\sqrt{N} n(t) .
$$

Our goal is to find the CRB for the estimation of $\{\rho, \psi, N\}$ based on our observation (2). In particular, the main focus is on the estimation of $\rho$ when $\psi, N$ are unknown. Previous works have addressed a related problem but based on the MF output sampled at the symbol rate [12], [25], [28].

The receive filter $g_{R}(t)$ is matched to the transmit pulse, i.e., $g_{R}(t)=g_{T}^{*}(-t)$. The MF output is denoted by $y(t)=$ $r(t) \star g_{R}(t)$. Then, within an observation interval of length $K T$, we can obtain $K$ of these symbol-rate MF output samples:

$$
\begin{aligned}
y_{k} & \triangleq y(k T)=\sqrt{\rho N} \mathrm{e}^{\jmath \psi} \sum_{l} a_{l} h((k-l) T)+\sqrt{N} w_{k} \\
& =\sqrt{\rho N} \mathrm{e}^{\jmath \psi} a_{k}+\sqrt{N} w_{k}, k \in\left\{k_{0}, \ldots, k_{0}+K-1\right\},
\end{aligned}
$$

where the Nyquist property of $h(t) \triangleq g_{T}(t) \star g_{R}(t)$ has been used $(h(n T)=1$ for $n=0$ and 0 otherwise); the noise samples are given by $w_{k} \triangleq w(k T)$ with $w(t)=n(t) \star g_{R}(t)$. Note that $\left\{w_{k}\right\}$ is zero-mean white Gaussian with unit variance.

\section{THE DUAL FILTER FRAMEWORK}

Since (3) is obtained by sampling $y(t)$ below its Nyquist rate $\frac{1+\alpha}{T}$, some useful information (for estimation purposes) may have been lost in the process. To avoid such loss, $r(t)$ could be oversampled, but this results in a sequence whose signal component is correlated; if $y(t)$ is oversampled instead, then the noise component becomes correlated as well. These correlations make the derivation of the $\mathrm{CRB}$ quite cumbersome. We adopt an alternative approach, described next.

\footnotetext{
${ }^{1}$ This is equivalent to the SNR per symbol, commonly denoted by $E_{s} / N_{0}$
}

\section{A. Development}

We choose to retain the symbol-rate MF output samples $y_{k}$ from (3), as well as the symbol-rate samples of the output of an auxiliary filter with impulse response $\widetilde{g}_{R}(t)$. As it turns out, by appropriate selection of the auxiliary filter, these two symbol-rate sequences preserve all information about $r(t)$. The impulse response $\widetilde{g}_{R}(t)$ is selected so that its convolution with the transmit pulse has zero crossings every $T$ seconds:

$$
\widetilde{h}(t)=g_{T}(t) \star \widetilde{g}_{R}(t) \quad \Rightarrow \quad \widetilde{h}(n T)=0 \quad \forall n \in \mathbb{Z} .
$$

Let us now define

$$
\widetilde{y}(t) \triangleq r(t) \star \widetilde{g}_{R}(t), \quad \widetilde{w}(t) \triangleq n(t) \star \widetilde{g}_{R}(t) .
$$

From (4), the auxiliary symbol-rate samples are given by

$$
\begin{aligned}
\widetilde{y}_{k} \triangleq \widetilde{y}(k T) & =\sqrt{\rho N} \mathrm{e}^{\jmath \psi} \sum_{l} a_{l} \widetilde{h}((k-l) T)+\sqrt{N} \widetilde{w}(k T) \\
& =\sqrt{N} \widetilde{w}_{k}, \quad k \in\left\{k_{0}, \ldots, k_{0}+K-1\right\}
\end{aligned}
$$

and thus contain only noise samples $\widetilde{w}_{k} \triangleq \widetilde{w}(k T)$. These are zero-mean Gaussian with autocorrelation $\mathrm{E}\left\{\widetilde{w}_{k} \widetilde{w}_{k-m}^{*}\right\}=$ $R_{\widetilde{w}}(m T)$, with $R_{\widetilde{w}}(\tau)$ the autocorrelation of $\widetilde{w}(t)$. Since $r(t)$ has no spectral content in $|f|>\frac{1+\alpha}{2 T}$, it can be assumed w.l.o.g. that $\widetilde{g}_{R}(\tau)$ is bandlimited to $\pm \frac{1+\alpha}{2 T}$, so that $R_{\widetilde{w}}(\tau)=$ $\widetilde{g}_{R}(\tau) \star \widetilde{g}_{R}^{*}(-\tau)$. The following result will be key in the sequel.

Lemma 1. If $\widetilde{g}_{R}(t)$ satisfies the zero-crossing property (4), then $\left\{w_{k}\right\}$ and $\left\{\widetilde{w}_{k}\right\}$ are uncorrelated: $\mathrm{E}\left\{w_{k} \widetilde{w}_{n}^{*}\right\}=0 \forall k, n$.

It follows from Lemma 1 that $y_{k}, \widetilde{y}_{n}$ are statistically independent $\forall k, n$ (since the noise is Gaussian and independent of data). This will greatly simplify the derivation of the CRB.

Characterizing all $\widetilde{h}(t)$ satisfying (4) was first addressed in [23], where the design of $\widetilde{g}_{R}(t)$ was undertaken in order to exploit the spectral coherence of the cyclostationary signal. An alternative description, valid for standard Nyquist pulses $h(t)$ such as the RC pulses considered here, is given next.

Lemma 2. Let $\widetilde{h}_{0}(t) \triangleq t \cdot h(t)$ have $F T \widetilde{H}_{0}(f)$. Then all filters $\widetilde{h}(t)$ satisfying the zero-crossing condition (4) are given in terms of their FT $\widetilde{H}(f)$ by

$$
\widetilde{H}(f)=P(f) \widetilde{H}_{0}(f), \quad|f| \leq \frac{1+\alpha}{2 T},
$$

where $P(f)$ is any transfer function satisfying

$$
P\left(f+\frac{1}{2 T}\right)=P\left(f-\frac{1}{2 T}\right), \quad|f| \leq \frac{\alpha}{2 T} .
$$

Note that if the inverse FT $p(t)$ of $P(f)$ is real-valued, then (8) amounts to $P\left(\frac{1}{2 T}+f\right)=P\left(\frac{1}{2 T}-f\right)$ for $|f| \leq \frac{\alpha}{2 T}$, so that $P(f)$ has Hermitian symmetry in the roll-off band.

Clearly, $P(f)=1$ meets $(8)$, and thus $\widetilde{H}(f)=\widetilde{H}_{0}(f)$ satisfies $^{2}$ (4). Since $H(f)=G_{T}^{2}(f)$, one has

$$
\widetilde{H}_{0}(f)=-\frac{1}{2 \pi \jmath} \frac{\mathrm{d} H(f)}{\mathrm{d} f}=-\frac{2 G_{T}(f)}{2 \pi \jmath} \frac{\mathrm{d} G_{T}(f)}{\mathrm{d} f} .
$$

Hence, since $\widetilde{H}(f)=G_{T}(f) \widetilde{G}_{R}(f)$, it follows from (7) and (9) that the $\widetilde{g}_{R}(t)$ resulting in the desired zero-crossing pattern (4) can be parameterized in the frequency domain by

$$
\widetilde{G}_{R}(f)=P(f) D(f), \quad \text { with } \quad D(f) \triangleq-\frac{1}{\pi j} \frac{\mathrm{d} G_{T}(f)}{\mathrm{d} f},
$$

\footnotetext{
${ }^{2}$ This is also evident by examining $\widetilde{h}_{0}(t)=t \cdot h(t)$, since $h(t)$ is Nyquist.
} 
for any $P(f)$ satisfying (8). We will refer to $\widetilde{G}_{R}(f)$ as Spectrum-Derivative Matched Filter (SDMF). Outside the rolloff band, $G_{T}(f)$ is flat and thus $D(f)=\widetilde{G}_{R}(f)=0$.

Non-differentiability of $G_{T}(f)$ at $f= \pm \frac{1+\alpha}{2 T}$ results in a sharp cutoff of $D(f)$ at these frequencies. Thus, in practice, an appropriate choice of $P(f)$ may ease SDMF design. When computing the $\mathrm{CRB}$, however, implementation issues are not relevant; it suffices to ensure that $\left\{y_{k}\right\},\left\{\widetilde{y}_{k}\right\}$ preserve all information about the analog input, as addressed next.

Lemma 3. Let $r(t)$ be bandlimited to $\pm \frac{1+\alpha}{2 T}$. Let $\widetilde{G}_{R}(f)$ be as in (10), with $P(f)$ satisfying (8). Then $r(t)$ can be recovered from $\left\{y_{k}\right\},\left\{\widetilde{y}_{k}\right\}$ iff $P(f) \neq 0$ for all $f$ in the roll-off band.

This is intuitively satisfying. By sampling the MF output at the symbol rate, aliasing occurs in the roll-off band only; to be able to compensate for this, the SDMF must not eliminate any content in this region. Thus, we will assume that $\widetilde{G}_{R}(f) \neq 0$ in the roll-off band, imposing w.l.o.g. the normalization

$$
\int_{-\infty}^{\infty} \widetilde{g}_{R}^{2}(t) \mathrm{d} t=1
$$

\section{B. Implications for CRB computation}

Let us focus now on the spectral characteristics of the SDMF output $\widetilde{y}_{k}=\widetilde{w}_{k}$, which is colored with autocorrelation $R_{\widetilde{w}}(m T)$. In particular, (11) implies $\mathrm{E}\left\{\left|\widetilde{w}_{k}\right|^{2}\right\}=R_{\widetilde{w}}(0)=1$. The following result states an important asymptotic property.

Lemma 4. Let $\widetilde{\boldsymbol{C}} \in \mathbb{C}^{K \times K}$ be the autocorrelation matrix of $\left\{\widetilde{w}_{k}\right\}$. As $K \rightarrow \infty$, one has $L \triangleq \operatorname{rank} \widetilde{\boldsymbol{C}} \rightarrow \alpha K$.

The fact that $\widetilde{\boldsymbol{C}}$ becomes (asymptotically) singular for $\alpha<1$ introduces a slight difficulty when computing the CRB, since the probability density function (pdf) of a Gaussian vector with a singular covariance matrix does not exist [29]. Consider, however, the following result [30, Lemma 1]:

Lemma 5. Let $\widetilde{\boldsymbol{w}}=\left[\begin{array}{ccc}\widetilde{w}_{1} & \cdots & \widetilde{w}_{K}\end{array}\right]^{T}$ and $\widetilde{\boldsymbol{C}}=\mathrm{E}\left\{\widetilde{\boldsymbol{w}} \widetilde{\boldsymbol{w}}^{H}\right\}$. Let $L \triangleq \operatorname{rank} \widetilde{\boldsymbol{C}} \leq K$, and let $\widetilde{\boldsymbol{C}}=\widetilde{\boldsymbol{U}} \widetilde{\boldsymbol{\Lambda}} \widetilde{\boldsymbol{U}}^{H}$, where $\widetilde{\boldsymbol{\Lambda}}$ is $L \times L$ diagonal with the $L$ nonzero eigenvalues, and $\widetilde{\boldsymbol{U}}$ is $K \times L$ with $\widetilde{\boldsymbol{U}}{ }^{H} \widetilde{\boldsymbol{U}}=\boldsymbol{I}_{L}$. Consider the random $L \times 1$ vector $\overline{\boldsymbol{v}} \triangleq \widetilde{\boldsymbol{\Lambda}}^{-1 / 2} \widetilde{\boldsymbol{U}}^{H} \widetilde{\boldsymbol{w}}$. Then it holds that $\widetilde{\boldsymbol{w}}=\widetilde{\boldsymbol{U}} \widetilde{\boldsymbol{\Lambda}}^{1 / 2} \overline{\boldsymbol{v}}$.

Thus, $\widetilde{\boldsymbol{w}} \in \mathbb{C}^{K}$ is statistically equivalent to $\widetilde{\boldsymbol{v}} \in \mathbb{C}^{L}$ (since there exists a one-to-one mapping between them, independent of unknown parameters). Hence, the CRB based on the symbol-rate sampled MF and SDMF outputs, collected in

$$
\begin{aligned}
& \boldsymbol{y}=\left[\begin{array}{llll}
y_{k_{0}} & y_{k_{0}+1} & \cdots & y_{k_{0}+K-1}
\end{array}\right]^{T}, \\
& \widetilde{\boldsymbol{y}}=\left[\begin{array}{llll}
\widetilde{y}_{k_{0}} & \widetilde{y}_{k_{0}+1} & \cdots & \widetilde{y}_{k_{0}+K-1}
\end{array}\right]^{T},
\end{aligned}
$$

is the same (for large $K$ ) as that based on $\boldsymbol{y}$ and on the vector

$$
\overline{\boldsymbol{y}} \triangleq \widetilde{\boldsymbol{\Lambda}}^{-1 / 2} \widetilde{\boldsymbol{U}}^{H} \widetilde{\boldsymbol{y}}=\sqrt{N} \overline{\boldsymbol{v}},
$$

since $\widetilde{\boldsymbol{y}}=\widetilde{\boldsymbol{U}} \widetilde{\boldsymbol{\Lambda}}^{1 / 2} \overline{\boldsymbol{y}}$ by Lemma 5 . Conditioned on $N, \overline{\boldsymbol{y}} \in \mathbb{C}^{L}$ is Gaussian distributed with full-rank covariance $\mathrm{E}\left\{\overline{\boldsymbol{y}} \bar{y}^{H}\right\}=$ $N \cdot \mathrm{E}\left\{\overline{\boldsymbol{v}} \overline{\boldsymbol{v}}^{H}\right\}=N \boldsymbol{I}_{L}$. In addition, $L=\alpha K$ by Lemma 4 .

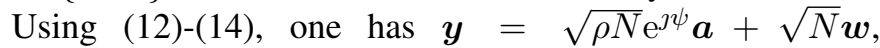
$\overline{\boldsymbol{y}}=\sqrt{N} \overline{\boldsymbol{v}}$, with $\boldsymbol{a}$ the vector of data symbols, and $\boldsymbol{w} \sim$
$\mathcal{C N}\left(\mathbf{0}, \boldsymbol{I}_{K}\right), \overline{\boldsymbol{v}} \sim \mathcal{C N}\left(\mathbf{0}, \boldsymbol{I}_{L}\right)$, with $\mathrm{E}\left\{\boldsymbol{w} \overline{\boldsymbol{v}}^{H}\right\}=\mathbf{0}_{K \times L}$. Letting $\boldsymbol{z} \triangleq\left[\boldsymbol{y}^{H} \overline{\boldsymbol{y}}^{H}\right]^{H}$, the observation model can be written as

$$
\boldsymbol{z}=\sqrt{\rho N} \mathrm{e}^{\jmath \psi}\left[\begin{array}{l}
\boldsymbol{a} \\
\mathbf{0}
\end{array}\right]+\sqrt{N}\left[\begin{array}{c}
\boldsymbol{w} \\
\overline{\boldsymbol{v}}
\end{array}\right] .
$$

We are now ready to use model (15) in order to derive estimation bounds. We will use the results from Lemmas 4 and 5, so that the results in the sequel are valid for large $K$.

\section{CRAmér-Rao Bound FOR The DA CASE}

Assume that the vector of symbols $\boldsymbol{a}$ is known (DA case), and normalized to $\frac{1}{K} \boldsymbol{a}^{H} \boldsymbol{a}=1$. Then $\boldsymbol{z} \sim \mathcal{C N}\left(\boldsymbol{\mu}_{z}, \boldsymbol{C}_{z}\right)$ with

$$
\boldsymbol{\mu}_{z}=\sqrt{\rho N} \mathrm{e}^{\jmath \psi}\left[\begin{array}{l}
\boldsymbol{a} \\
\mathbf{0}
\end{array}\right], \quad \boldsymbol{C}_{z}=N \boldsymbol{I}_{K+L} .
$$

Let $\boldsymbol{\theta}=[\psi \rho N]^{T}$ be the unknown parameter vector. The elements $\boldsymbol{J}_{\theta_{i} \theta_{j}}$ of the Fisher Information Matrix (FIM) $\boldsymbol{J}$ in the Gaussian case are given by the Slepian-Bangs formula [31, Eq. (B.3.25)], yielding $\boldsymbol{J}_{\psi \rho}=\boldsymbol{J}_{\psi N}=0$ when applied to (16). Hence, the parameter sets $\{\psi\}$ and $\{\rho, N\}$ are decoupled: the $\mathrm{CRB}$ for any of these sets is the same independently of whether the other is regarded as known or unknown. Thus, the CRB for phase offset, given by $\boldsymbol{J}_{\psi \psi}^{-1}=\frac{1}{2 \rho K}$ [32], is not improved by the availability of SDMF output samples. On the other hand, for large $K$, the $(\rho, N)$ subblock of the FIM is given by

$$
\left[\begin{array}{cc}
\boldsymbol{J}_{\rho \rho} & \boldsymbol{J}_{\rho N} \\
\boldsymbol{J}_{N \rho} & \boldsymbol{J}_{N N}
\end{array}\right]=\frac{K}{2}\left[\begin{array}{cc}
\frac{1}{\rho} & \frac{1}{N} \\
\frac{1}{N} & \frac{\rho+2(1+\alpha)}{N^{2}}
\end{array}\right] .
$$

From (17), the CRB for DA SNR estimation is given by

$$
\operatorname{CRB}(\rho)=\frac{1}{K} \rho\left(\frac{\rho}{1+\alpha}+2\right) .
$$

The corresponding bound when only the $K$ samples of the symbol-rate MF output are exploited is (see [9])

$$
\operatorname{CRB}_{\mathrm{MF}}(\rho)=\frac{1}{K} \rho(\rho+2) .
$$

The bounds (18)-(19) coincide for $\alpha=0$, since the symbolrate MF output is alias-free in that case, conveying all information about $r(t)$. As $\rho \rightarrow 0$, both bounds approach $\frac{2 \rho}{K}$ : in low SNR, the information contributed by the SDMF output becomes less significant. On the other hand, one has

$$
\lim _{\rho \rightarrow \infty} \frac{\operatorname{CRB}(\rho)}{\operatorname{CRB}_{\mathrm{MF}}(\rho)}=\frac{1}{1+\alpha} \in\left[\frac{1}{2}, 1\right],
$$

i.e., for high SNR the availability of the SDMF output reduces the DA bound up to $3 \mathrm{~dB}$, depending on excess bandwidth.

The DA CRB (18) can also be compared with that from [9, eq. (64)] based on the oversampled MF input with oversampling factor $N_{\mathrm{ss}}$ (in this way, the noise psd becomes $S_{n}(f)=1$ for $|f| \leq \frac{N_{\mathrm{ss}}}{2 T}$ and zero elsewhere), which is $\frac{1}{K} \rho\left(\frac{\rho}{N_{\mathrm{ss}}}+2\right)$. Both bounds coincide for $N_{\mathrm{ss}}=1+\alpha$, since in that case both models process the same bandwidth and become equivalent.

For completeness, the DA ML SNR estimator is derived next. The DA ML estimates of $\psi, S=\rho N$, and $N$ are

$$
\widehat{\psi}=\arg \left\{\boldsymbol{a}^{H} \boldsymbol{y}\right\}, \quad \widehat{S}=\frac{1}{K^{2}}\left|\boldsymbol{a}^{H} \boldsymbol{y}\right|^{2},
$$




$$
\widehat{N}=\frac{1}{1+\alpha}\left(\frac{\|\boldsymbol{y}\|^{2}}{K}-\widehat{S}\right)+\frac{\alpha}{1+\alpha}\left(\frac{\|\bar{y}\|^{2}}{\alpha K}\right) .
$$

Then $\widehat{\rho}=\frac{\widehat{S}}{\widehat{N}}$ by the invariance property [33, Th. 7.2]. From (21), availability of the SDMF output is irrelevant when estimating $\psi$ and $S$. On the other hand, such availability does improve the noise power estimate $\widehat{N}$, obtained as the convex combination of the estimates individually obtained from the MF (by subtracting the estimated signal power $\widehat{S}$ from the estimated total power) and SDMF outputs. The weights of this convex combination are determined by the excess bandwidth.

\section{CRAmÉR-RAo Bound FOR THE NDA CASE}

In the NDA case, (15) is still valid, but now $\boldsymbol{a}$ is zero-mean random with covariance $\boldsymbol{I}_{K}$, statistically independent of $\boldsymbol{w}$ and $\overline{\boldsymbol{v}}$. Thus, $\boldsymbol{y}$ and $\overline{\boldsymbol{y}}$ are statistically independent, so that the FIM based on $\boldsymbol{z}$ is the sum of the corresponding FIMs: $\boldsymbol{J}=\boldsymbol{J}^{\mathrm{MF}}+$ $\boldsymbol{J}^{\mathrm{SDMF}}$, where $\boldsymbol{J}^{\mathrm{MF}}$ is the FIM for the estimation problem based on $\boldsymbol{y}$ only, which was derived in [12], whereas $\boldsymbol{J}^{\mathrm{SDMF}}$ denotes the FIM based on $\overline{\boldsymbol{y}}$. Since $\overline{\boldsymbol{y}}$ does not depend on $\{\psi, \rho\}$, one has $\boldsymbol{J}^{\mathrm{SDMF}}=\operatorname{diag}\left\{\left[\begin{array}{lll}0 & 0 & \boldsymbol{J}_{N N}^{\mathrm{SDMF}}\end{array}\right]\right\}$. Since $\overline{\boldsymbol{y}}$ is Gaussian, $\boldsymbol{J}_{N N}^{\text {SDMF }}$ can be obtained from the Slepian-Bangs formula. Thus,

$$
\boldsymbol{J}_{N N}^{\mathrm{SDMF}}=\frac{K \alpha}{N^{2}} \quad \text { for large } K .
$$

In the NDA setting one has $\boldsymbol{J}_{\psi \rho}^{\mathrm{MF}}=\boldsymbol{J}_{\psi N}^{\mathrm{MF}}=0$ [12]. Hence, as in the DA case, availability of the SDMF output does not affect the CRB for $\psi$, given by $\boldsymbol{J}_{\psi \psi}^{-1}=\frac{F_{M}(\rho)}{2 \rho K}$, with the specific form of $F_{M}(\rho) \geq 1$ depending on the constellation [12], [32]. On the other hand, the CRB for NDA SNR estimation is

$$
\operatorname{CRB}(\rho)=\frac{\boldsymbol{J}_{N N}^{\mathrm{SDMF}}+\boldsymbol{J}_{N N}^{\mathrm{MF}}}{\boldsymbol{J}_{\rho \rho}^{\mathrm{MF}}\left(\boldsymbol{J}_{N N}^{\mathrm{SDMF}}+\boldsymbol{J}_{N N}^{\mathrm{MF}}\right)-\left(\boldsymbol{J}_{\rho N}^{\mathrm{MF}}\right)^{2}},
$$

to be compared against the corresponding CRB when only the $K$ symbol-rate samples at the MF output are available:

$$
\operatorname{CRB}_{\mathrm{MF}}(\rho)=\frac{\boldsymbol{J}_{N N}^{\mathrm{MF}}}{\boldsymbol{J}_{\rho \rho}^{\mathrm{MF}} \boldsymbol{J}_{N N}^{\mathrm{MF}}-\left(\boldsymbol{J}_{\rho N}^{\mathrm{MF}}\right)^{2}}
$$

In view of (23), the bounds (24)-(25) coincide if $\alpha=0$, as for the DA case. As noted in [26], $\boldsymbol{J}_{\rho \rho}^{\mathrm{MF}}$ is independent of $N$, whereas $\boldsymbol{J}_{\rho N}^{\mathrm{MF}} \propto \frac{1}{N}$ and $\boldsymbol{J}_{N N}^{\mathrm{MF}} \propto \frac{1}{N^{2}}$. Thus, the noise power $N$ cancels out in (25) so that $\mathrm{CRB}_{\mathrm{MF}}$ emerges as a function of $\rho$ only. In view of (23), the same is true of the CRB in (24).

In general, the bounds (24)-(25) must be evaluated numerically [26]-[28]. Next we consider several illustrative cases in which closed-form expressions can be obtained.

\section{A. Quadrature Phase-Shift Keying (QPSK) symbols}

For i.i.d. symbols drawn from a QPSK constellation, the CRB (25) has been derived in [24]. The $\{\rho, N\}$ subblock of the MF-based FIM can be written as

$$
\left[\begin{array}{cc}
\boldsymbol{J}_{\rho \rho}^{\mathrm{MF}} & \boldsymbol{J}_{\rho N}^{\mathrm{MF}} \\
\boldsymbol{J}_{N \rho}^{\mathrm{MF}} & \boldsymbol{J}_{N N}^{\mathrm{MF}}
\end{array}\right]=\frac{K}{N^{2}}\left[\begin{array}{cc}
\frac{N^{2}}{2 \rho}(1-g(\rho)) & \frac{N}{2}(1+g(\rho)) \\
\frac{N}{2}(1+g(\rho)) & 1+\frac{\rho}{2}(1-g(\rho))
\end{array}\right],
$$

where

$$
g(\rho) \triangleq \frac{\mathrm{e}^{-\rho / 2}}{\sqrt{2 \pi}} \int_{-\infty}^{\infty} \frac{u^{2} \mathrm{e}^{-u^{2} / 2}}{\cosh (u \sqrt{\rho})} \mathrm{d} u
$$

From these and (24)-(25), one finds that

$$
\begin{aligned}
\operatorname{CRB}(\rho) & =\frac{1}{K} \frac{2(1+\alpha) \rho+\rho^{2}(1-g(\rho))}{(1+\alpha)(1-g(\rho))-2 \rho g(\rho)}, \\
\operatorname{CRB}_{\mathrm{MF}}(\rho) & =\frac{1}{K} \frac{2 \rho+\rho^{2}(1-g(\rho))}{1-g(\rho)-2 \rho g(\rho)} .
\end{aligned}
$$

Since $\lim _{\rho \rightarrow \infty} g(\rho)=0$, the NDA bounds (27) and (28) tend to the corresponding DA bounds (18) and (19) respectively. Thus, for QPSK in the NDA case, (20) also holds. On the other hand, the third-order Taylor series approximation of $g(\rho)$ about $\rho=0$ is $g(\rho) \approx 1-2 \rho+4 \rho^{2}-10 \rho^{3}$. Using this, the CRBs (27) and (28) are found to behave respectively as $\frac{1}{K}\left(1+\frac{1}{\alpha}\right)$ and $\frac{1}{K \rho^{2}}$ for small $\rho$. Thus, in the low SNR regime, if excess bandwidth is not exploited, then the bound deteriorates much faster as $\rho \rightarrow 0$. In contrast, in the DA case both bounds behave as $\frac{2 \rho}{K}$ for small $\rho$ regardless of $\alpha$, as seen in Sec. IV.

\section{B. Gaussian symbols}

Although symbols are always drawn from a discrete, finite constellation in practice, the Gaussian distribution results in the largest CRB [34], so it is of interest to derive such bound. Thus, assume that $\boldsymbol{a} \sim \mathcal{C N}\left(\mathbf{0}, \boldsymbol{I}_{K}\right)$. The carrier phase $\psi$ cannot be estimated with Gaussian symbols but, in any case, the CRB for the estimation of $\{\rho, N\}$ is not sensitive to the unknown $\psi$, since these parameter sets are decoupled as observed earlier.

With Gaussian symbols, one has $\boldsymbol{y} \sim \mathcal{C N}\left(\mathbf{0}, N(\rho+1) \boldsymbol{I}_{K}\right)$. From the Slepian-Bangs formula, the MF-based FIM becomes

$$
\left[\begin{array}{ll}
\boldsymbol{J}_{\rho \rho}^{\mathrm{MF}} & \boldsymbol{J}_{\rho N}^{\mathrm{MF}} \\
\boldsymbol{J}_{N \rho}^{\mathrm{MF}} & \boldsymbol{J}_{N N}^{\mathrm{MF}}
\end{array}\right]=K\left[\begin{array}{cc}
\frac{1}{(\rho+1)^{2}} & \frac{1}{N(\rho+1)} \\
\frac{1}{N(\rho+1)} & \frac{1}{N^{2}}
\end{array}\right]
$$

which is singular, due to the pdf of $\boldsymbol{y}$ depending on $\rho, N$ exclusively through $(\rho+1) N$ : the pair $\{\rho, N\}$ is not identifiable from the symbol-rate MF output, and thus $\operatorname{CRB}_{\mathrm{MF}}(\rho)=$ $\infty$. However, once we substitute $\boldsymbol{J}_{N N}^{\mathrm{MF}}=\frac{K}{N^{2}}$ in (29) by $\boldsymbol{J}_{N N}^{\mathrm{MF}}+\boldsymbol{J}_{N N}^{\mathrm{SDMF}}=\frac{K}{N^{2}}+\frac{K \alpha}{N^{2}}=\frac{(1+\alpha) K}{N^{2}}$, the FIM becomes nonsingular and the $\mathrm{CRB}$ is readily obtained:

$$
\operatorname{CRB}(\rho)=\frac{1}{K}\left(1+\frac{1}{\alpha}\right)(\rho+1)^{2} .
$$

For high SNR, (30) behaves as $\frac{\rho^{2}}{K}\left(1+\frac{1}{\alpha}\right)$, whereas the corresponding bound (18) for the DA case behaves as $\frac{\rho^{2}}{K} \frac{1}{1+\alpha}$. Thus, in the high SNR region, the NDA-CRB for Gaussian symbols is larger than the DA-CRB by a factor $\left(2+\alpha+\frac{1}{\alpha}\right)$, which is always larger than 4 (i.e., $6 \mathrm{~dB}$ ). On the other hand, for $\rho \ll 1$, the bound (30) goes to $\frac{1}{K}\left(1+\frac{1}{\alpha}\right)$, which is the same value as for QPSK symbols (Sec. V-A). This suggests that, in the low SNR region, knowledge about the symbol constellation is not relevant as far as the NDA-CRB is concerned. This issue will be further developed in Sec. V-C.

Maximizing the log-likelihood $f(\boldsymbol{z} \mid S, N)$ and using $L=$ $\alpha K$, the ML estimates of $N, S$ and $\rho$ under the Gaussian symbol assumption are readily found:

$$
\widehat{N}=\frac{\|\bar{y}\|^{2}}{\alpha K}, \quad \widehat{S}=\frac{\|\boldsymbol{y}\|^{2}}{K}-\widehat{N}, \quad \widehat{\rho}=\frac{\widehat{S}}{\widehat{N}}=\alpha \frac{\|\boldsymbol{y}\|^{2}}{\|\overline{\boldsymbol{y}}\|^{2}}-1 .
$$

Note that $\widehat{N}$ is obtained as the sample variance of the SDMF output, without using $\boldsymbol{y}$. The signal power is estimated by subtracting $\widehat{N}$ from the sample variance of the MF output. 


\section{CRB for Quadratically Constrained Estimators}

The FIM for the general NDA estimation problem under the constraint that estimators use only second-order statistics of the data (i.e., quadratic functions of $\boldsymbol{z}$ ) was developed in [35]. We apply now those results to the SNR estimation problem.

Let $\boldsymbol{a}$ in (15) be a random vector of independent symbols drawn from an arbitrary zero-mean, unit-variance distribution with kurtosis $k_{4} \triangleq \mathrm{E}\left\{\left|a_{k}\right|^{4}\right\}-2$. The problem is that of estimating $\{\rho, N\}$ from quadratic transformations of the observations, collected in $\operatorname{vec}\left(\boldsymbol{y} \boldsymbol{y}^{H}\right)$ and $\operatorname{vec}\left(\overline{\boldsymbol{y}} \bar{y}^{H}\right)$. The cross-terms vec $\left(\overline{\boldsymbol{y}} \boldsymbol{y}^{H}\right)$, vec $\left(\boldsymbol{y} \overline{\boldsymbol{y}}^{H}\right)$ are not considered since they are zero-mean, due to statistical independence of $\boldsymbol{y}, \overline{\boldsymbol{y}}$. Furthermore, the terms vec $\left(\boldsymbol{y} \boldsymbol{y}^{T}\right)$, vec $\left(\overline{\boldsymbol{y}} \bar{y}^{T}\right)$ are zero-mean as well in general $^{3}$, and will not be taken into account either.

It turns out that the carrier phase is not identifiable by quadratic schemes [35]. Nevertheless, the bounds for the estimation of $\{\rho, N\}$ are not affected by lack of knowledge about $\psi$, analogously to the Gaussian case in Sec. V-B.

The global FIM is the sum of those based on vec $\left(\boldsymbol{y} \boldsymbol{y}^{H}\right)$ and vec $\left(\bar{y} \bar{y}^{H}\right)$, since these terms are statistically independent. Let us focus first on the FIM based on vec $\left(\boldsymbol{y} \boldsymbol{y}^{H}\right)$ alone, which can be derived using [35, eq. (53)]:

$$
\left[\begin{array}{cc}
\boldsymbol{J}_{\rho \rho}^{\mathrm{MF}} & \boldsymbol{J}_{\rho N}^{\mathrm{MF}} \\
\boldsymbol{J}_{N \rho}^{\mathrm{MF}} & \boldsymbol{J}_{N N}^{\mathrm{MF}}
\end{array}\right]=\left[\begin{array}{cc}
\frac{\partial \boldsymbol{m}^{H}}{\partial \rho} \boldsymbol{Q}^{-1} \frac{\partial \boldsymbol{m}}{\partial \rho} & \frac{\partial \boldsymbol{m}^{H}}{\partial \rho} \boldsymbol{Q}^{-1} \frac{\partial \boldsymbol{m}}{\partial N} \\
\frac{\partial \boldsymbol{m}^{H}}{\partial N} \boldsymbol{Q}^{-1} \frac{\partial \boldsymbol{m}}{\partial \rho} & \frac{\partial \boldsymbol{m}^{H}}{\partial N} \boldsymbol{Q}^{-1} \frac{\partial \boldsymbol{m}}{\partial N}
\end{array}\right],
$$

where $\boldsymbol{m}$ and $\boldsymbol{Q}$ are respectively the mean and covariance of $\operatorname{vec}\left(\boldsymbol{y} \boldsymbol{y}^{H}\right)$, which can be computed using [35, eq. (34)]:

$$
\begin{aligned}
\boldsymbol{m} & \triangleq \mathrm{E}\left\{\operatorname{vec}\left(\boldsymbol{y} \boldsymbol{y}^{H}\right)\right\}=(\rho+1) N \operatorname{vec}\left(\boldsymbol{I}_{K}\right), \\
\boldsymbol{Q} & \triangleq \mathrm{E}\left\{\operatorname{vec}\left(\boldsymbol{y} \boldsymbol{y}^{H}\right) \operatorname{vec}^{H}\left(\boldsymbol{y} \boldsymbol{y}^{H}\right)\right\}-\boldsymbol{m} \boldsymbol{m}^{H} \\
& =(\rho+1)^{2} N^{2} \boldsymbol{I}_{K^{2}}+k_{4} \rho^{2} N^{2} \operatorname{diag}\left\{\operatorname{vec}\left(\boldsymbol{I}_{K}\right)\right\} .
\end{aligned}
$$

After some algebra, (32) is found to be

$$
\left[\begin{array}{ll}
\boldsymbol{J}_{\rho \rho}^{\mathrm{MF}} & \boldsymbol{J}_{\rho N}^{\mathrm{MF}} \\
\boldsymbol{J}_{N \rho}^{\mathrm{MF}} & \boldsymbol{J}_{N N}^{\mathrm{MF}}
\end{array}\right]=\frac{K}{(\rho+1)^{2}+\rho^{2} k_{4}}\left[\begin{array}{cc}
1 & \frac{\rho+1}{N} \\
\frac{\rho+1}{N} & \frac{(\rho+1)^{2}}{N^{2}}
\end{array}\right],
$$

which is singular, meaning that the SNR cannot be estimated quadratically from the symbol-rate MF output samples, regardless of the constellation; the SDMF output must be also processed. The FIM based on vec $\left(\overline{\boldsymbol{y}} \overline{\boldsymbol{y}}^{H}\right)$ is [35, eq. (53)]:

$$
\left[\begin{array}{ll}
\boldsymbol{J}_{\rho \rho}^{\mathrm{SDMF}} & \boldsymbol{J}_{\rho N}^{\mathrm{SDMF}} \\
\boldsymbol{J}_{N \rho}^{\mathrm{SDMF}} & \boldsymbol{J}_{N N}^{\mathrm{SDMF}}
\end{array}\right]=\left[\begin{array}{cc}
\frac{\partial \overline{\boldsymbol{m}}^{H}}{\partial \rho} \overline{\boldsymbol{Q}}^{-1} \frac{\partial \overline{\boldsymbol{m}}}{\partial \rho} & \frac{\partial \overline{\boldsymbol{m}}^{H}}{\partial \rho} \overline{\boldsymbol{Q}}^{-1} \frac{\partial \overline{\boldsymbol{m}}}{\partial N} \\
\frac{\partial \overline{\boldsymbol{m}}^{H}}{\partial N} \overline{\boldsymbol{Q}}^{-1} \frac{\partial \overline{\boldsymbol{m}}}{\partial \rho} & \frac{\partial \overline{\boldsymbol{m}}^{H}}{\partial N} \overline{\boldsymbol{Q}}^{-1} \frac{\partial \overline{\boldsymbol{m}}}{\partial N}
\end{array}\right]
$$

where now $\overline{\boldsymbol{m}}$ and $\overline{\boldsymbol{Q}}$ stand for the mean and covariance of $\operatorname{vec}\left(\overline{\boldsymbol{y}} \overline{\boldsymbol{y}}^{H}\right)$, which, using [35, eq. (34)], are found to be

$$
\begin{aligned}
& \overline{\boldsymbol{m}} \triangleq \mathrm{E}\left\{\operatorname{vec}\left(\overline{\boldsymbol{y}} \overline{\boldsymbol{y}}^{H}\right)\right\}=N \operatorname{vec}\left(\boldsymbol{I}_{L}\right), \\
& \overline{\boldsymbol{Q}} \triangleq \mathrm{E}\left\{\operatorname{vec}\left(\overline{\boldsymbol{y}} \overline{\boldsymbol{y}}^{H}\right) \operatorname{vec}^{H}\left(\overline{\boldsymbol{y}} \overline{\boldsymbol{y}}^{H}\right)\right\}-\overline{\boldsymbol{m}} \overline{\boldsymbol{m}}^{H}=N^{2} \boldsymbol{I}_{L^{2}} .
\end{aligned}
$$

Using (37)-(38), the only nonzero entry in (36) turns out to be $\boldsymbol{J}_{N N}^{\text {SDMF }}=\frac{\alpha K}{N^{2}}$. Hence, from the global FIM $\boldsymbol{J}^{\mathrm{MF}}+\boldsymbol{J}^{\text {SDMF }}$ the CRB for quadratic SNR estimators is found:

$$
\operatorname{CRB}(\rho)=\frac{1}{K}\left(1+\frac{1}{\alpha}\right)(\rho+1)^{2}+\frac{1}{K} k_{4} \rho^{2},
$$

\footnotetext{
${ }^{3}$ Exceptions to this general rule can be found when using certain modulation formats such as Binary Phase-Shift Keying (BPSK), offset QPSK (O-QPSK), or Minimum Shift Keying (MSK), which will not be considered in this paper.
}

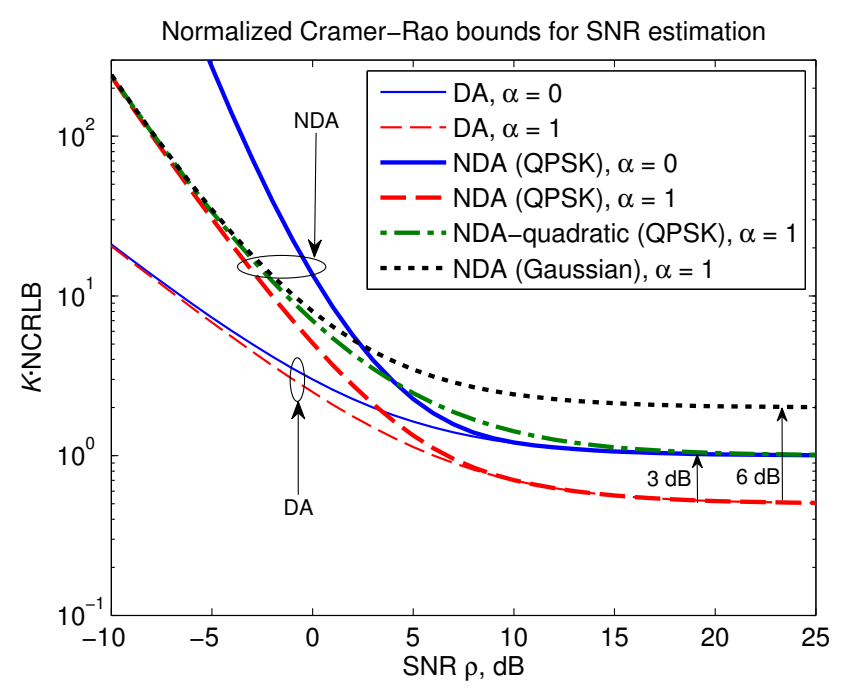

Fig. 1. CRBs for SNR estimation under different models.

which reduces to (30) for Gaussian symbols $\left(k_{4}=0\right)$, since second-order statistics convey all information under Gaussian observations. In practice, constellations are sub-Gaussian (or platykurtic), i.e., $k_{4}<0$ : the Gaussian symbols assumption constitutes a "worst case" for quadratic estimators. Constantmodulus (CM) constellations have the smallest possible kurtosis $k_{4}=-1$, yielding the lowest quadratic CRB (39). Note that, as $\rho \rightarrow 0$, (39) approaches $\frac{1}{K}\left(1+\frac{1}{\alpha}\right)$, the same value as for the unconstrained bounds for QPSK and Gaussian symbols.

The CRB (39) is achievable by practical quadratic estimators. The following result applies to the Noise Subspace Estimator (NSE) from [23], which was derived based on a dual filter framework similar to the one presented in Sec. III:

$$
\widehat{\rho}=\frac{\|\boldsymbol{y}\|^{2}}{\|\widetilde{\boldsymbol{y}}\|^{2}}-1 \text {. }
$$

Lemma 6. Let $\widetilde{G}_{R}(f)=P(f) D(f)$ with $P(f)$ chosen as

$$
P(f)=\left\{\begin{array}{cc}
\jmath c \frac{G_{T}\left(f-\frac{1}{T}\right)}{D(f)}, & \frac{1-\alpha}{2 T}<f<\frac{1+\alpha}{2 T}, \\
-\jmath c \frac{G_{T}\left(f+\frac{1}{T}\right)}{D(f)}, & -\frac{1+\alpha}{2 T}<f<-\frac{1-\alpha}{2 T},
\end{array}\right.
$$

and zero otherwise, with $D(f)$ as in (10) and c a normalization constant to ensure (11). Then, as $K \rightarrow \infty$, the NSE (40) approaches the ML estimator for Gaussian symbols (31), and its asymptotic variance approaches the quadratic CRB (39).

Thus, with a suitable choice of the SDMF $\widetilde{G}_{R}(f)$, the NSE asymptotically becomes optimal among the class of quadratic estimators, regardless of the symbol constellation. We remark that under (41), the psd of the symbol-rate SDMF output becomes flat within the rolloff band, which is the key for NSE optimality (see Appendix E). Another feature of (40) is that the colored SDMF output samples are directly incorporated in the estimator, thus avoiding whitening steps such as (14).

\section{NUMERICAL EXAMPLES}

Focusing on QPSK, Fig. 1 shows the normalized bound $\operatorname{NCRB}(\rho) \triangleq \operatorname{CRB}(\rho) / \rho^{2}$ for the two extreme cases $\alpha=0$ and $\alpha=1$. The following observations can be made: 
(a) 16-QAM

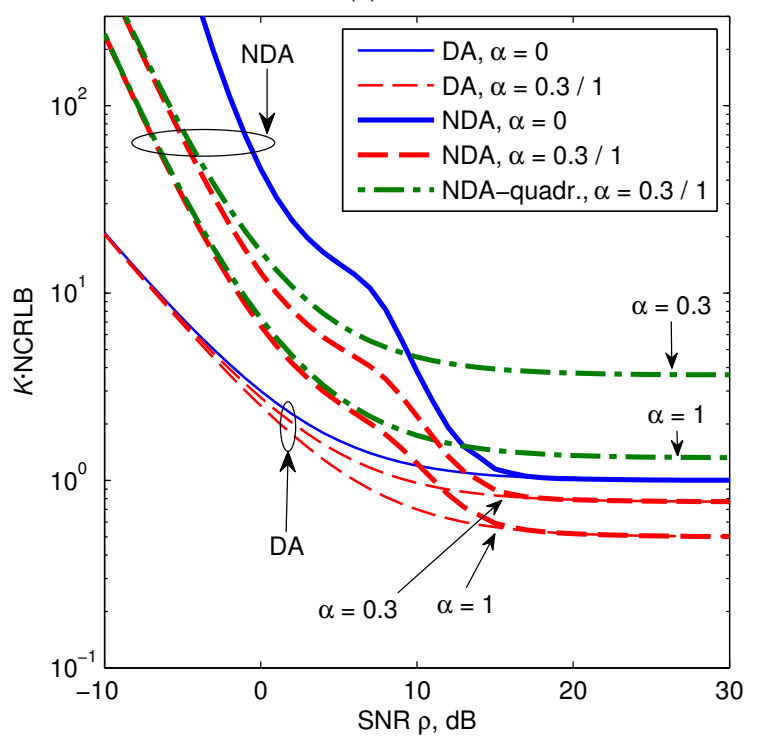

(b) 64-QAM

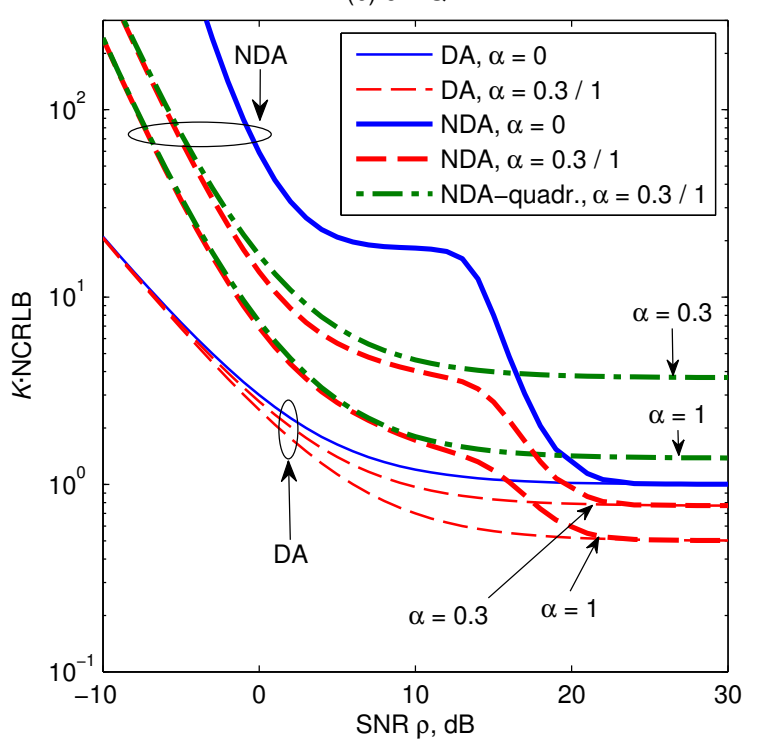

Fig. 2. CRBs for SNR estimation under different models with non-CM constellations: (a) 16-QAM, (b) 64-QAM

- For $\alpha=0$ (no excess bandwidth) the DA and NDA CRBs coincide with the known MF-based bounds from [26]. Note that for $\alpha=0$, the NDA CRBs corresponding to Gaussian symbols (30) or to quadratic estimators (39) go to infinity, and thus they are not depicted in Fig. 1.

- The DA bounds for $\alpha=0$ and $\alpha=1$ converge for low SNR, so that in this regime the information contributed by the SDMF output become less relevant. On the other hand, in high SNR the availability of such samples results in a sizable improvement of the CRB (of up to $3 \mathrm{~dB}$ for $\alpha=1$ ).

- The QPSK NDA bounds converge to their DA counterparts in high SNR. This was observed in [26] for the MF-based bounds $(\alpha=0)$, and is as expected, since in this region decision-directed operation becomes feasible.

- In low SNR, the NDA bounds for QPSK and Gaussian symbols exploiting MF and SDMF output samples converge. Hence, as the SNR goes to zero, knowledge about the constellation becomes irrelevant for SNR estimation purposes.

- In high SNR, the NDA CRB for Gaussian symbols is larger than that for QPSK (6 dB for $\alpha=1$ ). For multilevel constellations (QAM, APSK) the NDA CRB will lie between those for QPSK and Gaussian symbols. As long as the constellation is discrete, the NDA CRB will eventually converge to the DA CRB if the SNR is sufficiently high.

- The quadratic constraint on NDA estimators degrades the CRB in high SNR. With $\alpha=1$, this loss is up to $3 \mathrm{~dB}$ for $\mathrm{CM}$ constellations, and will approach $6 \mathrm{~dB}$ for very dense multilevel constellations. In low SNR, the quadratic and unconstrained NDA bounds coincide.

Fig. 2 shows the bounds for 16- and 64-QAM. For these non-CM constellations (with kurtoses $k_{4}=-0.68$ and -0.619 respectively), the gap in high SNR between the quadratic and unconstrained NDA CRBs is larger than for QPSK (Fig. 1). For low SNR these bounds still converge, indicating that in this regime second-order statistics convey all relevant information (as long as $\alpha>0$ ), regardless of the underlying constellation.
With denser constellations, the point at which the unconstrained NDA bounds touch their DA counterparts is progressively shifted to higher SNR values. As a result, a "mediumSNR" region opens up, over which the benefit of exploiting the excess bandwidth becomes larger; for example, with 64QAM at $10 \mathrm{~dB}$ SNR the corresponding CRB is 6.6 and $10 \mathrm{~dB}$ lower, for $\alpha=0.3$ and $\alpha=1$ respectively, than for $\alpha=0$.

\section{CONCLUSIONS}

With the aid of the dual filter framework, it has been shown that exploiting available excess bandwidth improves the CRB for SNR estimation, in an amount that depends on the roll-off factor in different ways for different bound types, and which can be significant with non-CM constellations. The developed bounds are independent of the auxiliary filter, as long as it fullfils certain perfect reconstruction property ensuring that no information about the analog signal is lost due to sampling. The ML DA and optimal quadratic NDA estimators have also been given. Although irrelevant for the derivation of bounds, the fact that $D(f)$ is not continuous at the band edges raises implementation issues, which may be alleviated by appropriate choice of $P(f)$. Obtaining efficient estimators with practical choices of $P(f)$ is an open issue and the object of further work; similar tradeoffs have been observed in [23].

\section{APPENDIX}

\section{A. Proof of Lemma 1}

Let $R_{w \widetilde{w}}(\tau) \triangleq \mathrm{E}\left\{w(t) \widetilde{w}^{*}(t-\tau)\right\}$ and $R_{n}(\tau) \triangleq$ $\mathrm{E}\left\{n(t) n^{*}(t-\tau)\right\}$. Then $R_{w \widetilde{w}}(\tau)=R_{n}(\tau) \star \widetilde{g}_{R}^{*}(-\tau) \star g_{R}(\tau)=$ $R_{n}(\tau) \star \widetilde{h}^{*}(-\tau)=\widetilde{h}^{*}(-\tau)$, where the last step follows from the fact that $g_{T}(t)$, and therefore $\widetilde{h}^{*}(-t)$ as well, is bandlimited to $\pm \frac{1+\alpha}{2 T}$, whereas the FT of $R_{n}(\tau)$ is $S_{n}(f)=1$ for $|f| \leq \frac{1+\alpha}{2 T}$ and 0 otherwise. Using the zero-crossing property (4), it follows that $\mathrm{E}\left\{w_{k} \widetilde{w}_{n}^{*}\right\}=R_{w \widetilde{w}}((k-n) T)=$ $h^{*}((n-k) T)=0$ for all $k, n$. 


\section{B. Proof of Lemma 2}

The requirements in (4) can be rewritten as

$\widetilde{h}(t) \cdot \sum_{n} \delta(t-n T)=0 \Leftrightarrow \frac{1}{T} \sum_{n} \widetilde{H}\left(f-\frac{n}{T}\right)=0$,

where $\widetilde{H}(f)=G_{T}(f) \widetilde{G}_{R}(f)$ is the FT of $\widetilde{h}(t)$, which is bandlimited to $\pm \frac{1+\alpha}{2 T}$. By $(42), \widetilde{H}(f)$ must be zero for $|f|<\frac{1-\alpha}{2 T}$. In the roll-off band only two replicas overlap, so that $\stackrel{H}{H}(f)+\widetilde{H}\left(f-\frac{1}{T}\right)=0$ for $\frac{1-\alpha}{2 T} \leq f \leq \frac{1+\alpha}{2 T}$, i.e.,

$$
\widetilde{H}\left(f-\frac{1}{2 T}\right)+\widetilde{H}\left(f+\frac{1}{2 T}\right)=0, \quad|f| \leq \frac{\alpha}{2 T} .
$$

Let $P(f)$ satisfy (8). Then it is readily seen that $P(f) \widetilde{H}(f)$ is also a solution to $(43)$. On the other hand, let $\widetilde{H}_{1}(f), \widetilde{H}_{2}(f)$ satisfy (43), with $\widetilde{H}_{2}(f) \neq 0$ for $\frac{1-\alpha}{2 T} \leq f \leq \frac{1+\alpha}{2 T}$. Then let

$$
P(f)=\frac{\widetilde{H}_{1}(f)}{\widetilde{H}_{2}(f)}, \quad \frac{1-\alpha}{2 T} \leq f \leq \frac{1+\alpha}{2 T},
$$

so that $\widetilde{H}_{1}(f)=P(f) \widetilde{H}_{2}(f)$ in the roll-off band. In addition,

$$
\begin{aligned}
0 & =\widetilde{H}_{1}\left(f-\frac{1}{2 T}\right)+\widetilde{H}_{1}\left(f+\frac{1}{2 T}\right) \\
& =P\left(f-\frac{1}{2 T}\right) \widetilde{H}_{2}\left(f-\frac{1}{2 T}\right)+P\left(f+\frac{1}{2 T}\right) \widetilde{H}_{2}\left(f+\frac{1}{2 T}\right) \\
& =\left[P\left(f-\frac{1}{2 T}\right)-P\left(f+\frac{1}{2 T}\right)\right] \widetilde{H}_{2}\left(f-\frac{1}{2 T}\right),
\end{aligned}
$$

for $|f| \leq \frac{\alpha}{2 T}$, yielding $P\left(f-\frac{1}{2 T}\right)-P\left(f+\frac{1}{2 T}\right)=0,|f| \leq \frac{\alpha}{2 T}$.

Hence, all solutions to (43) can be written as $\widetilde{H}(f)=$ $P(f) \widetilde{H}_{0}(f)$ with $P(f)$ satisfying $(8)$ and $\widetilde{H}_{0}(f)$ a particular solution not vanishing in the roll-off band. Clearly, $\widetilde{h}_{0}(t) \triangleq$ $t \cdot h(t)$ has zero crossings at the specified time instants $t=n T$, $n \in \mathbb{Z}$, and it is readily checked that its FT

$$
\widetilde{H}_{0}(f)=-\frac{1}{2 \pi \jmath} \frac{\mathrm{d} H(f)}{\mathrm{d} f}=-\frac{1}{2 \pi \jmath} \frac{\mathrm{d}\left|G_{T}(f)\right|^{2}}{\mathrm{~d} f}
$$

is nonzero in $\frac{1-\alpha}{2 T} \leq|f| \leq \frac{1+\alpha}{2 T}$ for the considered pulses.

\section{Proof of Lemma 3}

Consider the reconstruction of $r(t)$ by means of a pair of synthesis filters $c(t) \leftrightarrow C(f), \widetilde{c}(t) \leftrightarrow \widetilde{C}(f)$ :

$$
\hat{r}(t)=\sum_{k} y(k T) c(t-k T)+\sum_{k} \widetilde{y}(k T) \widetilde{c}(t-k T) .
$$

Taking Fourier transforms,

$$
\begin{aligned}
\hat{R}(f)= & \frac{1}{T}\left[\sum_{n} G_{R}\left(f-\frac{n}{T}\right) R\left(f-\frac{n}{T}\right)\right] C(f) \\
& +\frac{1}{T}\left[\sum_{n} \widetilde{G}_{R}\left(f-\frac{n}{T}\right) R\left(f-\frac{n}{T}\right)\right] \widetilde{C}(f) .
\end{aligned}
$$

Since $R(f)=0$ for $|f|>\frac{1+\alpha}{2 T}$, we can rewrite (48) as

$$
\begin{aligned}
\hat{R}(f)= & \frac{1}{T}\left[G_{R}(f) C(f)+\widetilde{G}_{R}(f) \widetilde{C}(f)\right] R(f) \\
& +\frac{1}{T}\left[G_{R}\left(f-\frac{1}{T}\right) C(f)+\widetilde{G}_{R}\left(f-\frac{1}{T}\right) \widetilde{C}(f)\right] \\
& \times R\left(f-\frac{1}{T}\right), \quad 0<f \leq \frac{1+\alpha}{2 T}, \\
\hat{R}(f)= & \frac{1}{T}\left[G_{R}(f) C(f)+\widetilde{G}_{R}(f) \widetilde{C}(f)\right] R(f) \\
& +\frac{1}{T}\left[G_{R}\left(f+\frac{1}{T}\right) C(f)+\widetilde{G}_{R}\left(f+\frac{1}{T}\right) \widetilde{C}(f)\right] \\
& \times R\left(f+\frac{1}{T}\right), \quad-\frac{1+\alpha}{2 T} \leq f \leq 0 .
\end{aligned}
$$

Hence, it suffices to find synthesis filters satisfying

$$
\begin{aligned}
& {\left[\begin{array}{cc}
G_{R}(f) & \widetilde{G}_{R}(f) \\
G_{R}\left(f-\frac{1}{T}\right) & \widetilde{G}_{R}\left(f-\frac{1}{T}\right)
\end{array}\right]\left[\begin{array}{l}
C(f) \\
\widetilde{C}(f)
\end{array}\right]=\left[\begin{array}{c}
T \\
0
\end{array}\right], f \in I^{+},} \\
& {\left[\begin{array}{cc}
G_{R}(f) & \widetilde{G}_{R}(f) \\
G_{R}\left(f+\frac{1}{T}\right) & \widetilde{G}_{R}\left(f+\frac{1}{T}\right)
\end{array}\right]\left[\begin{array}{l}
C(f) \\
\widetilde{C}(f)
\end{array}\right]=\left[\begin{array}{c}
T \\
0
\end{array}\right], f \in I^{-},}
\end{aligned}
$$

where $I^{+} \triangleq\left[0, \frac{1+\alpha}{2 T}\right], I^{-} \triangleq\left[-\frac{1+\alpha}{2 T}, 0\right]$. Assume that $P(f) \neq 0$ for all $f$ in the roll-off band, and consider the filters

$$
C(f)=G_{R}(f), \quad \widetilde{C}(f)=\left\{\begin{array}{cc}
\frac{T-\left|G_{R}(f)\right|^{2}}{\widetilde{G}_{R}(f)}, & \frac{1-\alpha}{2 T} \leq|f| \leq \frac{1+\alpha}{2 T} \\
0, & \text { otherwise }
\end{array}\right.
$$

Note that $\widetilde{C}(f)$ is well defined, since $P(f) \neq 0 \Rightarrow \widetilde{G}_{R}(f) \neq$ 0 in the roll-off band. It can be readily shown that if $P(f)$ satisfies (8), then (53) constitutes a valid solution to (51)-(52); the proof is straightforward and thus it is omitted for brevity.

Finally, if $P\left(f_{1}\right)=0$ for some $f_{1}$ in the roll-off band, then $P\left(f_{1}-\frac{1}{T}\right)=0$ from (8). Hence $\widetilde{G}_{R}\left(f_{1}\right)=\widetilde{G}_{R}\left(f_{1}-\frac{1}{T}\right)=0$, and the conditions (51) at $f=f_{1}$ read as $G_{R}\left(f_{1}\right) C\left(f_{1}\right)=T$, $G_{R}\left(f_{1}-\frac{1}{T}\right) C\left(f_{1}\right)=0$. But there exists no $C\left(f_{1}\right)$ satisfying these, since $G_{R}\left(f_{1}\right) \neq 0$ and $G_{R}\left(f_{1}-\frac{1}{T}\right) \neq 0$.

\section{Proof of Lemma 4}

One has $\mathrm{E}\left\{\widetilde{w}_{k} \widetilde{w}_{n}^{*}\right\}_{\widetilde{G}}=R_{\widetilde{w}}((k-n) T)$, with $R_{\widetilde{w}}(\tau)=$ $\left[\widetilde{g}_{R}(\tau) \star \widetilde{g}_{R}^{*}(-\tau)\right] \leftrightarrow\left|\widetilde{G}_{R}(f)\right|^{2}$. Thus, the psd of $\left\{\widetilde{w}_{k}\right\}$ is

$$
S_{\widetilde{w}}\left(\mathrm{e}^{\jmath 2 \pi f T}\right)=\frac{1}{T} \sum_{n}\left|\widetilde{G}_{R}\left(f-\frac{n}{T}\right)\right|^{2} .
$$

Note that $\left|\widetilde{G}_{R}(f)\right|^{2}=0$ outside the roll-off band. Hence,

$$
S_{\widetilde{w}}\left(\mathrm{e}^{\jmath 2 \pi f T}\right)=0 \quad \text { for } \quad|f|<\frac{1-\alpha}{2 T} .
$$

Let $\lambda_{0}, \lambda_{1}, \ldots, \lambda_{K-1}$ be the eigenvalues of the $K \times K$ autocorrelation matrix $\widetilde{\boldsymbol{C}}$ of the process $\left\{\widetilde{w}_{k}\right\}$. It is well known [36], [37] that, since $\widetilde{\boldsymbol{C}}$ is Hermitian Toeplitz, these eigenvalues can be approximated as $K \rightarrow \infty$ as follows:

$$
\lambda_{k} \rightarrow S_{\widetilde{w}}\left(\mathrm{e}^{\jmath \frac{2 \pi k}{K}}\right), \quad 0 \leq k \leq K-1 .
$$

From (55) and (56) it follows that $\widetilde{\boldsymbol{C}}$ becomes rank deficient for large $K$. Moreover, if we let $L=\operatorname{rank} \widetilde{\boldsymbol{C}}$, i.e., the number of nonzero eigenvalues, it is seen from (55) and the $2 \pi$ periodicity of $S_{\widetilde{w}}\left(\mathrm{e}^{\jmath \omega}\right)$ that $\frac{L}{K} \rightarrow \frac{\alpha / T}{1 / T}=\alpha$ for $K \rightarrow \infty$.

\section{E. Proof of Lemma 6}

First we show that $P(f)$ in (41) is a valid filter, i.e., it satisfies (8). Note that for $P(f)$ as in (41), one has

$$
P\left(f \pm \frac{1}{2 T}\right)= \pm \jmath c \frac{G_{T}\left(f \mp \frac{1}{2 T}\right)}{D\left(f \pm \frac{1}{2 T}\right)}, \quad|f| \leq \frac{\alpha}{2 T} .
$$

To proceed, note that since $G_{T}(f)$ is bandlimited to $\pm \frac{1+\alpha}{2 T}$ and $\left|G_{T}(f)\right|^{2}$ is the spectrum of a Nyquist pulse, it holds that

$$
\left|G_{T}\left(f-\frac{1}{2 T}\right)\right|^{2}+\left|G_{T}\left(f+\frac{1}{2 T}\right)\right|^{2}=T, \quad|f| \leq \frac{\alpha}{2 T} .
$$

Recalling the definition of $D(f)$ from (10), and since $G_{T}(f)$ is real and nonnegative, taking derivatives in (58) one obtains $G_{T}\left(f-\frac{1}{2 T}\right) D\left(f-\frac{1}{2 T}\right)+G_{T}\left(f+\frac{1}{2 T}\right) D\left(f+\frac{1}{2 T}\right)=0$ 
for $|f| \leq \frac{\alpha}{2 T}$, yielding (8) in view of (57); thus, the symbolrate sampled SDMF output blocks the signal component.

The next step is to find the psd $S_{\widetilde{w}}\left(\mathrm{e}^{j 2 \pi f T}\right)$ of $\widetilde{w}_{k}$, given by (54). Note that for the chosen $P(f)$ in (41),

$$
\widetilde{G}_{R}(f)=\left\{\begin{array}{cc}
\jmath c G_{T}\left(f-\frac{1}{T}\right), & \frac{1-\alpha}{2 T}<f<\frac{1+\alpha}{2 T}, \\
-\jmath c G_{T}\left(f+\frac{1}{T}\right), & -\frac{1+\alpha}{2 T}<f<-\frac{1-\alpha}{2 T}, \\
0, & \text { otherwise. }
\end{array}\right.
$$

Thus, only two replicas of $\left|\widetilde{G}_{R}(f)\right|^{2}$ overlap in the rolloff band, so that for $\frac{1-\alpha}{2 T}<f<\frac{1+\alpha}{2 T}$,

$$
\begin{aligned}
S_{\widetilde{w}}\left(\mathrm{e}^{\jmath 2 \pi f T}\right) & =\frac{1}{T}\left[\left|\widetilde{G}_{R}(f)\right|^{2}+\left|\widetilde{G}_{R}\left(f-\frac{1}{T}\right)\right|^{2}\right] \\
& =\frac{c^{2}}{T}\left[\left|G_{T}\left(f-\frac{1}{T}\right)\right|^{2}+\left|G_{T}(f)\right|^{2}\right]=c^{2},(60)
\end{aligned}
$$

where the last step is due to the Nyquist property (58). On the other hand, $S_{\widetilde{w}}\left(\mathrm{e}^{j 2 \pi f T}\right)$ is zero outside the roll-off band, and since (11) amounts to $\frac{1}{2 \pi} \int_{0}^{2 \pi} S_{\widetilde{w}}\left(\mathrm{e}^{\jmath \omega}\right) \mathrm{d} \omega=1$, it follows that $c^{2}=\frac{1}{\alpha}$. Hence, the $L=\alpha K$ nonzero eigenvalues of $\widetilde{\boldsymbol{C}}$ are all equal (asymptotically) to $\frac{1}{\alpha}$, see (56), i.e., $\widetilde{\boldsymbol{\Lambda}} \rightarrow \frac{1}{\alpha} \boldsymbol{I}_{L}$ in the eigendecomposition $\widetilde{\boldsymbol{C}}=\widetilde{\boldsymbol{U}} \widetilde{\boldsymbol{\Lambda}} \widetilde{\boldsymbol{U}}^{H}$. From Lemma 5 and (14), $\widetilde{\boldsymbol{y}}=\widetilde{\boldsymbol{U}} \tilde{\boldsymbol{\Lambda}}^{1 / 2} \overline{\boldsymbol{y}}=\frac{1}{\sqrt{\alpha}} \widetilde{\boldsymbol{U}} \overline{\boldsymbol{y}}$ as $K \rightarrow \infty$, and thus $\|\widetilde{\boldsymbol{y}}\|^{2}=\frac{1}{\alpha} \overline{\boldsymbol{y}}^{H} \widetilde{\boldsymbol{U}}^{H} \widetilde{\boldsymbol{U}} \overline{\boldsymbol{y}}=\frac{1}{\alpha}\|\overline{\boldsymbol{y}}\|^{2}$. Substituting this in the NSE (40), one obtains the ML estimator for Gaussian symbols (31). The asymptotic variance of (40), given in [23, eq. (57)] (and noting that the choice of $P(f)$ in (41) yields $\gamma=1$ in that expression), is seen to coincide with the quadratic CRB (39).

\section{REFERENCES}

[1] U. Mengali and A. N. D'Andrea, Synchronization Techniques for Digital Receivers. New York: Plenum Press, 1997.

[2] H. Meyr, M. Moeneclaey, and S. A. Fechtel, Digital Communication Receivers: Synchronization, Channel Estimation, and Signal Processing. New York: Wiley-Interscience, 1997.

[3] R. M. Gagliardi and C. M. Thomas, "PCM data reliability monitoring through estimation of signal-to-noise ratio," IEEE Trans. Commun., vol. 16, pp. 479-486, Jun. 1968.

[4] T. A. Summers and S. G. Wilson, "SNR mismatch and online estimation in turbo decoding," IEEE Trans. Commun., vol. 46, pp. 421-423, Apr. 1998 .

[5] K. Balachandran, S. R. Kadaba, and S. Nanda, "Channel quality estimation and rate adaptation for cellular mobile radio," IEEE J. Sel. Areas Commun., vol. 17, pp. 1244-1256, Jul. 1999.

[6] T. S. Chung and A. Goldsmith, "Degrees of freedom in adaptive modulation: a unified view," IEEE Trans. Commun., vol. 49, pp. 15611571, Sep. 2001

[7] M. Mohammad and R. M. Buehrer, "On the impact of SNR estimation error on adaptive modulation," IEEE Commun. Lett., vol. 9, pp. 490-492, Jun. 2005.

[8] S. Cioni, R. De Gaudenzi, and R. Rinaldo, "Channel estimation and physical layer adaptation techniques for satellite networks exploiting adaptive coding and modulation," Int. J. Satell. Commun. Network., vol. 26, pp. 157-188, 2008.

[9] D. R. Pauluzzi and N. C. Beaulieu, "A comparison of SNR estimation techniques for the AWGN channel," IEEE Trans. Commun., vol. 48, pp. 1681-1691, Oct. 2000.

[10] W. Gappmair, R. López-Valcarce, and C. Mosquera, "ML and EM algorithm for non-data-aided SNR estimation of linearly modulated signals," in Proc. IEEE 6th Int. Symp. Communication Systems, Networks and Digital Signal Processing, Graz, Austria, Jul. 2008, pp. 530-534.

[11] A. Das, "NDA SNR estimation: CRLBs and EM based estimators," in Proc. IEEE Region 10 Conf., Hyderabad, India, Nov. 2008.

[12] W. Gappmair, R. López-Valcarce, and C. Mosquera, "Joint NDA estimation of carrier frequency/phase and SNR for linearly modulated signals," IEEE Signal Process. Lett., vol. 17, pp. 517-520, May 2010.
[13] E. Stathakis, J. Jaldén, L. K. Rasmussen, and M. Skoglund, "Uniformly improving Maximum-Likelihood SNR estimation of known signals in Gaussian channels," IEEE Trans. Signal Process., vol. 62, pp. 156-167, Jan. 2014

[14] T. Benedict and T. Soong, "The joint estimation of signal and noise from the sum envelope," IEEE Trans. Inf. Theory, vol. 13, pp. 447-454, Jul. 1968.

[15] R. Matzner, "An SNR estimation algorithm for complex baseband signals using higher order statistics," Facta Universitatis (Niš), vol. 1, pp. 41-52, 1993.

[16] P. Gao and C. Tepedelenlioğlu, "SNR estimation for nonconstant modulus constellations,” IEEE Trans. Signal Process., vol. 53, pp. 865-870, Mar. 2005.

[17] R. López-Valcarce and C. Mosquera, "Sixth-order statistics-based nondata-aided SNR estimation,” IEEE Commun. Lett., vol. 11, pp. 351-353, Apr. 2007.

[18] M. Álvarez-Díaz, R. López-Valcarce, and C. Mosquera, "SNR estimation for Multilevel Constellations Using Higher-Order Moments," IEEE Trans. Signal Process., vol. 58, pp. 1515-1526, Mar. 2010.

[19] A. Stéphenne, F. Bellili, and S. Affes, "Moment-based SNR estimation over linearly-modulated wireless SIMO channels," IEEE Trans. Wireless Commun., vol. 9, pp. 714-722, Feb. 2010.

[20] M. A. Boujelben, F. Bellili, S. Affes, and A. Stéphenne, "SNR estimation over SIMO channels from linearly modulated signals," IEEE Trans. Signal Process., vol. 58, pp. 6017-6028, Dec. 2010.

[21] A. Das and B. D. Rao, "SNR and noise variance estimation for MIMO systems," IEEE Trans. Signal Process., vol. 60, pp. 3929-3941, Aug. 2012.

[22] J. F. Sevillano, I. Velez, M. Leyh, S. Lipp, A. Irizar, and L. Fontan, "In-service SNR estimation without symbol timing recovery for QPSK data transmission systems ," IEEE Trans. Wireless Commun., vol. 6, pp. 3202-3207, Sep. 2007.

[23] J. Riba, J. Villares, and G. Vázquez, "A Nondata-Aided SNR estimation technique for multilevel modulations exploiting signal cyclostationarity," IEEE Trans. Signal Process., vol. 58, pp. 5767-5778, Nov. 2010.

[24] N. S. Alagha, "Cramér-Rao bounds of SNR estimates for BPSK and QPSK modulated signals," IEEE Commun. Lett., vol. 5, pp. 10-12, May 2001

[25] J.-P. Delmas, "Closed-form expressions of the exact Cramér-Rao bound for parameter estimation of BPSK, MSK, or QPSK waveforms," IEEE Signal Process. Lett., vol. 15, pp. 405-408, 2008.

[26] W. Gappmair, "Cramér-Rao lower bound for non-data-aided SNR estimation of linear modulation schemes," IEEE Trans. Commun., vol. 56, pp. 689-693, May 2008.

[27] W. Gappmair, R. López-Valcarce, and C. Mosquera, "Cramér-Rao lower bound and EM algorithm for envelope-based SNR estimation of nonconstant modulus constellations," IEEE Trans. Commun., vol. 57, pp. 1622-1627, Jun. 2009.

[28] F. Bellili, A. Stéphenne, and S. Affes, "Cramér-Rao Lower Bounds for NDA SNR Estimates of Square QAM Modulated Transmissions," IEEE Trans. Commun., vol. 58, pp. 3211-3218, Nov. 2010.

[29] R. M. Gray and L. D. Davisson, An Introduction to Statistical Signal Processing. Cambridge, UK: Cambridge University Press, 2010.

[30] J. F. Schmidt, D. Romero, and R. Lopez-Valcarce, "Active Interference Cancellation for OFDM Spectrum Sculpting: Linear Processing Is Optimal," IEEE Commun. Lett., vol. 18, pp. 1543-1546, Sep. 2014.

[31] P. Stoica and R. L. Moses, Spectral Analysis of Signals. Upper Saddle River, NJ: Prentice-Hall, 2005.

[32] F. Rice, B. Cowley, B. Moran, and M. Rice, "Cramér-Rao lower bounds for QAM phase and frequency estimation," IEEE Trans. Commun., vol. 49, pp. 1582-1591, Sep. 2001.

[33] S. M. Kay, Fundamentals of Statistical Signal Processing: Estimation Theory. Upper Saddle River, NJ: Prentice-Hall, 1993.

[34] P. Stoica and P. Babu, "The Gaussian data assumption leads to the largest Cramér-Rao bound,' IEEE Signal Process. Mag., vol. 28, pp. 132-133, May 2011.

[35] J. Villares and G. Vázquez, "Second-Order Parameter Estimation," IEEE Trans. Signal Process., vol. 53, pp. 2408-2420, Jul. 2005.

[36] S. M. Kay, Fundamentals of Statistical Signal Processing: Detection Theory. Englewood Cliffs, NJ: Prentice-Hall, 1998.

[37] R. M. Gray, Toeplitz and Circulant Matrices: a Review. New York: Hanover/Now, 2006. 


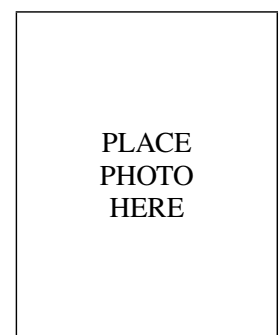

Roberto López-Valcarce (S'95-M'01) received the Telecommunication Engineering degree from the University of Vigo, Vigo, Spain, in 1995, and the M.S. and Ph.D. degrees in electrical engineering from the University of Iowa, Iowa City, in 1998 and 2000, respectively. During 1995 he was a Project Engineer with Intelsis. He was a Ramón y Cajal Postdoctoral Fellow of the Spanish Ministry of Science and Technology from 2001 to 2006. During that period, he was with the Signal Theory and Communications Department, Universidad de Vigo,

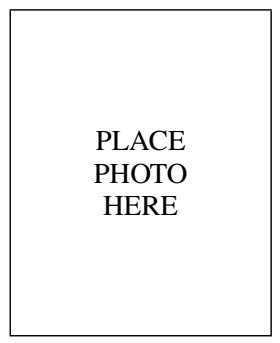

Carlos Mosquera where he currently is an Associate Professor. His main research interests lie in the area of adaptive signal processing, digital communications, and sensor networks, and has coauthored over 50 papers in leading international journals. He holds several patents in collaboration with industry.

Dr. López-Valcarce was a recipient of a 2005 Best Paper Award of the IEEE Signal Processing Society. He served as an Associate Editor of the IEEE TRANSACTIONS ON SIGNAL PROCESSING from 2008 to 2011, and as a member of the IEEE Signal Processing for Communications and Networking Technical Committee from 2011 to 2013.

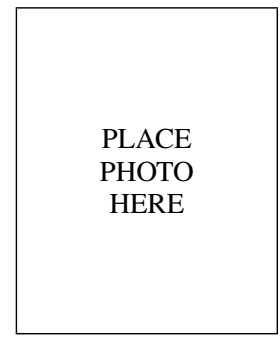

\section{Javier Villares}

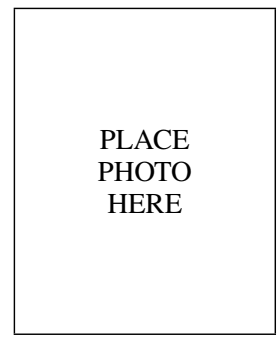

Jaume Riba

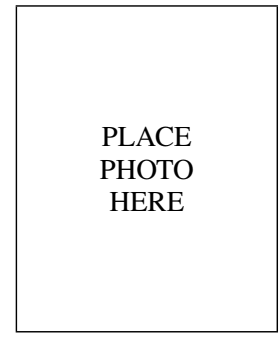

Wilfried Gappmair 